\title{
Benefícios das práticas religiosas na assistência de enfermagem: Uma revisão integrativa
}

\author{
Benefits of religious practices in nursing care: An integrative review \\ Beneficios de las prácticas religiosas en el cuidado de enfermería: Una revisión integradora
}

Recebido: 21/06/2021 | Revisado: 28/06/2021 | Aceito: 09/07/2021 | Publicado: 20/07/2021

Italo Lucas Lima Araujo

ORCID: https://orcid.org/0000-0002-4007-4122 Centro Universitário de Ciências e Tecnologia do Maranhão, Brasil

E-mail: italolimaaraujo25@hotmail.com

Chrisllayne Oliveira da Silva

ORCID: https://orcid.org/0000-0002-0844-0268

Universidade Federal do Piauí, Brasil

E-mail: chris-layne10@hotmail.com

Laila da Silva Mota Vilanova

ORCID: https://orcid.org/0000-0001-8873-5914

Centro Universitário de Ciências e Tecnologia do Maranhão, Brasil

E-mail: lailajoseneto@gmail.com

Amanda Thais Franco Oliveira

ORCID: https://orcid.org/0000-0002-4210-7485

Centro Universitário de Ciências e Tecnologia do Maranhão, Brasil

E-mail: https://orcid.org/0000-0002-4210-7485

Linccon Fricks Hernandes

ORCID: https://orcid.org/0000-0002-7642-3080 Escola Superior de Ciências da Santa Casa de Misericórdia de Vitória, Brasil E-mail: fricksjr@hotmail.com

Valeria Silva Carvalho

ORCID: https://orcid.org/0000-0003-1356-5804 Centro Universitário de Ciências e Tecnologia do Maranhão, Brasil E-mail: Valeriapsico28@gmail.com

Ilmara Patrícia Compasso da Silva de Queiroz ORCID: https://orcid.org/0000-0003-3348-0568 Centro Universitário de Ciências e Tecnologia do Maranhão, Brasil

E-mail: ilmaracompasso2014@outlook.com

Lisianne Natália Santos Silva

ORCID: https://orcid.org/0000-0002-2574-399X

Centro Universitário de Ciências e Tecnologia do Maranhão, Brasil E-mail: lisyanenathalia6@gmail.com

Ielda Pereira Rodrigues

ORCID: https://orcid.org/0000-0003-3365-4791

Instituto Athena de Educação, Brasil

E-mail: ieldapereira@hotmail.com

Ianeska Bárbara Ribeiro do Nascimento

ORCID: https://orcid.org/0000-0002-5319-289X

Centro Universitário de Ciências e Tecnologia do Maranhão, Brasil

E-mail: ianeska@outlook.com

Lucas Sousa Guimarães

ORCID: https://orcid.org/0000-0002-9228-8114

Centro Universitário de Ciências e Tecnologia do Maranhão, Brasil

E-mail: lucasguimaraes0310@gmail.com

Juliana de Sousa Oliveira Ximenes Cruz

ORCID: https://orcid.org/0000-0003-3430-171X

Centro Universitário de Ciências e tecnologia do Maranhão, Brasil

E-mail: xjuliana865@gmail.com

Lia Marinho da Silva Queiroz ORCID: https://orcid.org/0000-0002-5437-6877

Centro Universitário de Ciências e tecnologia do Maranhão, Brasil

E-mail: liamarinho1998@gmail.com

Solygardia Albuquerque Maciel Teixeira

ORCID: https://orcid.org/0000-0003-0214-0982

Centro Universitário Nossa Senhora do Patrocino, Brasi

E-mail: solygardia@bol.com.br 


\author{
Rafael Andrade da Silva \\ ORCID: https://orcid.org/0000-0002-0357-8102 \\ Centro Universitário de Ciências e Tecnologia do Maranhão, Brasil \\ E-mail: rafael98enfermeiro@gmail.com \\ Paulo Sérgio Gaspar dos Santos \\ ORCID: https://orcid.org/0000-0003-1763-642X \\ Centro Universitário de Ciências e Tecnologia do Maranhão, Brasil \\ E-mail: institutogasph@gmail.com
}

\begin{abstract}
Resumo
Desde os primórdios da ciência do cuidado ao ser humano, as práticas religiosas e a espiritualidade enfrentam um olhar rigoroso dos cientistas e pesquisadores. A assistência de enfermagem deve prestar cuidados e medidas de segurança ao indivíduo sob um modelo de unidade biopsicossocial, em todos os aspectos físicos, sociais, econômicos, espirituais e culturais. Nesse caminho, este artigo teve como objetivo geral analisar as produções científicas acerca dos benefícios das práticas religiosas na prestação de cuidados de enfermagem, e especificamente descrever a importância das práticas religiosas no estado de saúde, destacar as principais limitações e os desafios nos cuidados de enfermagem com as práticas de cura cultural-religiosa e relacionar as condutas de enfermagem com o tratamento e/ou terapia cultural/religiosa. Trata-se de uma revisão integrativa da literatura, na qual se formulou a questão: "Quais os benefícios da associação entre as práticas religiosas e assistência de enfermagem na prestação de cuidados?". Com uso de descritores e palavras-chaves, foram consultadas as bases de dados Bireme, CINAHL e PubMed. Utilizou-se os estudos disponíveis em totalidade, publicados nos anos de 2015 até 2020, nos idiomas Inglês, Português e Espanhol. Os artigos apresentaram que religiosidade e espiritualidade são dimensões fundamentais nos processos de cura, reabilitacionais e de superação, e devem ser implementadas na ciência do cuidado pelos profissionais de saúde. Concluiu-se que é dever da enfermagem e de outras áreas da saúde, atender o indivíduo com assistência humanizada, respeitando suas necessidades espiritual-religiosas no tratamento e/ou terapia.
\end{abstract}

Palavras-chave: Espiritualidade; Cuidados de enfermagem; Benefícios.

\begin{abstract}
Since the beginnings of the science of caring for human beings, religious practices and spirituality face a rigorous look from scientists and researchers. Nursing care must provide care and safety measures to the individual under a biopsychosocial unit model, in all physical, social, economic, spiritual and cultural aspects. In this way, this article aimed to analyze the scientific productions about the benefits of religious practices in the provision of nursing care, and specifically describe the importance of religious practices in health status, highlight the main limitations and challenges in nursing care with cultural-religious healing practices and relating nursing behavior to cultural/religious treatment and/or therapy. This is an integrative literature review, in which the question was formulated: "What are the benefits of the association between religious practices and nursing care in the provision of care?". Using descriptors and keywords, the Bireme, CINAHL and PubMed databases were consulted. We used the available studies in full, published in the years 2015 to 2020, in English, Portuguese and Spanish. The articles showed that religiosity and spirituality are fundamental dimensions in the healing, rehabilitation and overcoming processes, and should be implemented in the science of care by health professionals. It was concluded that it is the duty of nursing and other areas of health to serve the individual with humanized care, respecting their spiritual-religious needs in treatment and/or therapy.
\end{abstract}

Keywords: Spirituality; Nursing care; Benefits.

\title{
Resumen
}

Desde los inicios de la ciencia del cuidado del ser humano, las prácticas religiosas y la espiritualidad enfrentan una mirada rigurosa por parte de científicos e investigadores. El cuidado de enfermería debe brindar medidas de cuidado y seguridad al individuo bajo un modelo de unidad biopsicosocial, en todos los aspectos físicos, sociales, económicos, espirituales y culturales. De esta manera, este artículo tuvo como objetivo analizar las producciones científicas acerca de los beneficios de las prácticas religiosas en la prestación de cuidados de enfermería, y específicamente describir la importancia de las prácticas religiosas en el estado de salud, resaltar las principales limitaciones y desafíos en el cuidado de enfermería con los cuidados culturales-religiosos. prácticas curativas y relacionar el comportamiento de enfermería con el tratamiento y / o terapia cultural / religiosa. Se trata de una revisión integradora de la literatura, en la que se formuló la pregunta: "¿Cuáles son los beneficios de la asociación entre prácticas religiosas y cuidados de enfermería en la prestación de cuidados?". Mediante descriptores y palabras clave se consultaron las bases de datos Bireme, CINAHL y PubMed. Se utilizaron los estudios disponibles en su totalidad, publicados en los años 2015 a 2020 , en inglés, portugués y español. Los artículos mostraron que la religiosidad y la espiritualidad son dimensiones fundamentales en los procesos de curación, rehabilitación y superación, y deben ser implementadas en la ciencia del cuidado por los profesionales de la salud. Se concluyó que es deber de la enfermería y otras áreas de la salud atender al individuo con cuidados humanizados, respetando sus necesidades espiritual-religiosas en el tratamiento y / o terapia.

Palabras clave: Espiritualidad; Cuidado de enfermera; Beneficios. 


\section{Introdução}

O cuidado ao ser humano apresenta inúmeras abordagens ao decorrer dos séculos, tendo em sua concepção uma prática mecanicista ao cuidado. Atualmente, com a Polícia Nacional de Humanização o cuidado tem como foco integrado ao usuário, considerando diferentes dimensões do processo saúde-doença, e a espiritualidade precisa ser implantada no atendimento humanizado, já que é vista como uma das dimensões da subjetividade humana (Ferreira, Duarte, Silva, \& Bezerra, 2015).

O ser humano deve ser visto como uma unidade biopsicossocial sendo necessário que a equipe de saúde compreenda também a dimensão espiritual do cliente. Nesse contexto é importante que os enfermeiros compreendam o significado de religiosidade e espiritualidade na vida do paciente e da família (Cervelin \& Kruse, 2014). Além disso, a implementação dos termos espiritualidade e religiosidade quando exercida na prática, influenciam de forma direta a própria espiritualidade e religiosidade da equipe de enfermagem (Nascimento et al., 2013).

Em perspectiva, a espiritualidade e a religiosidade são dimensões do ser humano que podem modificar todo o estilo de vida do indivíduo, sendo observados aspectos positivos de mudança de hábitos, aceitação ao tratamento, fortalecimento e apoio emocional. A espiritualidade engloba fenômenos, domínio existencial e a essência do seu eu, fornecendo significado para a vida, enquanto a religiosidade é caracterizada por um conjunto de valores, regras e crenças em um poder ou ser superior (Oliveira et al., 2017).

Nos cuidados direcionados a patologias graves, com o medo frequente da morte, e que causam sofrimento ao paciente e a família, os cuidadores buscam estratégias de apoio que ofereçam um conforto e os ajudem a enfrentar a situação. Nestes casos, a religiosidade e a espiritualidade são usadas como mecanismos para o enfrentamento, superação e reabilitação do paciente (Alves et al., 2016).

No decorrer dos anos, a religiosidade vem ganhando espaço nos estudos sobre saúde, já que, por muito tempo, foi vista através de olhares críticos no meio científico. A religiosidade e espiritualidade têm correlação com a qualidade de vida, sendo um dos alicerces para o enfrentamento de certas patologias. É importante ressaltar que a espiritualidade e religiosidade devem ser usadas nas práticas de saúde incluídas nas formações profissionais (Melo, Sampaio, Souza, \& Pinto, 2015).

Nesse caminho, este estudo tem com questão norteadora: Quais os benefícios da associação entre práticas religiosas e assistência de enfermagem na prestação de cuidados? Tendo como objetivo geral do estudo analisar as produções científicas acerca dos benefícios das práticas religiosas na prestação de cuidados de enfermagem, e especificamente descrever a importância das práticas religiosas no estado de saúde, destacar as principais limitações e os desafios nos cuidados de enfermagem com as práticas de cura cultural-religiosa e relacionar as condutas de enfermagem com o tratamento e/ou terapia cultural/religiosa.

Na mídia, são relatadas muitas notícias sobre situações que ferem a saúde do cliente e/ou a morte de usuários pela falta de conhecimento, valores e respeito ao contexto sociocultural daqueles que utilizam o SUS. Na maior parte das vezes, profissionais que atuam na área de saúde simplesmente ignoram a crença e o status sociocultural do paciente.

Esses fatores de riscos ocorrem comumente pela falta de diálogo com os pacientes. Assim, a relevância desse estudo é ampliar a visão dos profissionais de saúde sobre a terapêutica assistencial combinada com a prática religiosa e espiritual de forma que seja eficaz, e que ao mesmo tempo respeite os princípios éticos e religiosos do paciente.

Destarte, o estudo tem como finalidade contribuir para o conhecimento dos profissionais de saúde sobre tratamentos e recursos terapêuticos provindos do agente de curas religiosas, estabelecendo um conhecimento construtivo de modo que o enfermeiro empregue seus cuidados e estabeleça medidas de segurança e avaliação ao paciente como unidade biopsicosociocultural. 


\section{Metodologia}

Realizou-se uma pesquisa bibliográfica do tipo revisão integrativa da literatura, um método que sintetiza os resultados de pesquisas anteriores sobre um determinado tema. Essa síntese de resultados permite comparar as informações existentes sobre um fenômeno obtendo conclusões, ou identificando a necessidade de se produzir mais estudos na área (Crossetti, 2012). Esse procedimento foi escolhido por ser capaz de viabilizar a sistematização de conhecimento científico, quando o pesquisador realiza a síntese e análise do conhecimento científico na evolução do tema. Esta revisão integrativa da literatura utilizou a metodologia proposta no estudo de Oliveira et al. (2016) para nortear as etapas metodológicas desta pesquisa.

Conforme Ercole, Melo e Alcoforado (2014), a revisão integrativa viabiliza a capacidade da sistematização do conhecimento científico, aproximando o pesquisador do problema abordado, possibilitando ser traçado um panorama sobre todas as produções científicas existentes e a possibilidade de desenvolver novos estudos na área de investigação.

Além disso, permite organizar e gerenciar conhecimento atual sobre um assunto, e aplicá-lo à prática clínica, uma vez que utiliza um rigor metodológico durante todas as etapas da revisão, possibilitando identificar as características dos estudos analisados, principais resultados, e qualidade de evidências (Pompeo, Rossi \& Galvão, 2009).

O tema "Benefícios das práticas religiosas na assistência de enfermagem", determinou a construção da estratégia PICo, que representa um acrônimo para Paciente (P), Interesse (I), e Contexto (Co), utilizada para a geração da questão norteadora desta revisão integrativa da literatura: "Quais os benefícios da associação entre práticas religiosas e assistência de enfermagem na prestação de cuidados?".

Para a localização dos estudos relevantes, que respondessem à pergunta de pesquisa, utilizou-se de descritores indexados e não indexados (palavras-chave) nos idiomas português, inglês e espanhol. Os descritores foram obtidos a partir do Medical Subject Headings (MESH), dos Descritores em Ciências da Saúde (DeCS) e dos títulos da Cumulative Index to Nursing and Allied Health Literature (CINAHL), como mostra o Quadro 01.

Consultou-se por meio de descritores e palavras-chave as bases de dados PubMed da National Library of Medicine; BVS (Biblioteca Virtual da Saúde), coordenada pela BIREME e composta de bases de dados bibliográficas produzidas pela Rede BVS, como LILACS, além da base de dados Medline, CINAHL e outros tipos de fontes de informação, sendo utilizado na busca o operador lógico de pesquisa “AND”.

Quadro 1. Elementos da estratégia PICO, descritores e palavras-chave utilizados - Caxias, MA, Brasil, 2020.

\begin{tabular}{|c|c|c|c|c|c|}
\hline & ELEMENTOS & DECS & MESH & $\begin{array}{l}\text { TÍTULOS } \\
\text { CINAHL }\end{array}$ & $\begin{array}{c}\text { PALAVRAS- } \\
\text { CHAVES }\end{array}$ \\
\hline $\mathbf{P}$ & "Práticas religiosas" & $\begin{array}{l}\text { "Religion” } \\
\text { "Religión" } \\
\text { "Religião" }\end{array}$ & "Religion" & "Religion" & - \\
\hline I & "Benefícios" & - & - & - & "Benefits" \\
\hline Co & $\begin{array}{l}\text { "Cuidados de } \\
\text { Enfermagem" }\end{array}$ & $\begin{array}{c}\text { "Nursing care" } \\
\text { “Atención de enfermaría", } \\
\text { "Cuidados de enfermagem" }\end{array}$ & "Nursingcare" & "Nursingcare" & - \\
\hline
\end{tabular}

Fonte: Decs, Cinahl, Mesh terms (2020).

Como critérios de inclusão, utilizou-se estudos disponíveis na íntegra, publicados nos últimos cinco anos (2015 até 2020), nos idiomas Inglês, Português e Espanhol. Foram excluídos da estratégia de busca textos incompletos, relatos técnicos, 
capítulo de livros, monografias, resumos e publicações que não abordassem a espiritualidade e religiosidade no âmbito da saúde.

A análise para seleção dos estudos foi realizada em duas fases, a saber: na primeira, os estudos foram pré-selecionados segundo os critérios de inclusão e exclusão, e de acordo com a estratégia de funcionamento e busca de cada base de dados.

Encontrou-se setenta e quatro (74) estudos como busca geral na BVS, sendo que limitando a busca para artigos com texto completo realizado com humanos nos últimos cinco anos, obteve-se quatorze (14) estudos, destes foram analisados títulos e resumos onde apenas seis (06) estudos foram condizentes com a questão desta pesquisa.

Na base PUBMED, como busca total, foram encontrados trinta (30) estudos. Aplicando-se na pesquisa o filtro que limita por texto completo dos últimos cinco anos com humanos, vinte e nove (29) estudos, foram analisados em seus títulos e resumos, e chegando-se ao resultado final de três (03) estudos.

Na base CINAHL, como busca total foram encontrados vinte e seis (26) estudos. Aplicando-se na pesquisa o filtro que limita por texto completo dos últimos cinco anos com humanos, um (01) estudo, analisado em seu título e resumo, sendo selecionado ao final.

$\mathrm{Na}$ análise e interpretação dos resultados, foram analisadas as informações dos artigos científicos selecionados e criadas categorias analíticas com base nos objetivos propostos no estudo, em seguida foi feita a sumarização e ordenação dos mesmos. Essa sumarização e categorização foram realizadas de forma descritiva, obtendo as informações mais relevantes para o estudo.

A pesquisa respeitou as citações dos estudos segundo seus autores, os conceitos, as definições, e ideias, e considerou os aspectos éticos nos artigos científicos incluídos na revisão integrativa. Optou-se pela análise de forma estatística e de texto, os dados também foram organizados em quadros e tabelas para facilitar a visualização e compreensão dos resultados.

As evidências científicas foram classificadas segundo os níveis e graus de recomendação propostos por Bork (2011), como mostra a Figura 1 a seguir:

Figura 1. Níveis de evidência e graus de recomendação - Caxias, MA, Brasil, 2020.

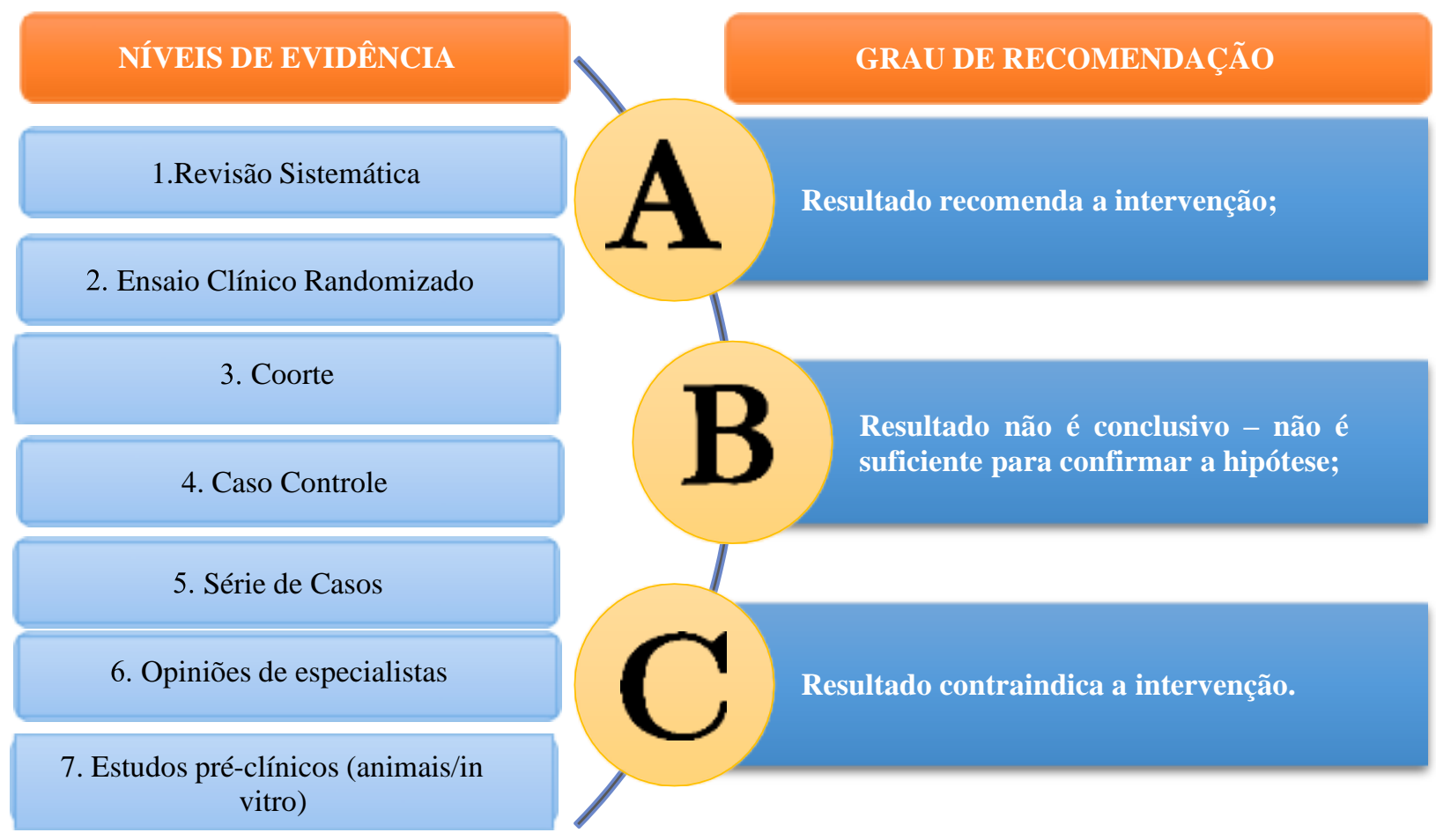

Fonte: Adaptado de Bork (2011). 


\section{Resultados}

A apresentação dos resultados está organizada em duas partes, sendo a primeira parte correspondente a caracterização dos estudos, e, a segunda parte relaciona-se ao cumprimento do objetivo do estudo, que diz respeito à análise da produção científica acerca dos benefícios das práticas religiosas na assistência de enfermagem.

Tabela 1. Análise descritiva das produções científicas acerca dos benefícios das práticas religiosas na assistência deenfermagem. Caxias - MA, 2020. $(\mathrm{N}=10)$.

\begin{tabular}{|c|c|c|}
\hline VARIÁVEIS & $\mathbf{N}$ & $\%$ \\
\hline \multicolumn{3}{|l|}{ Abordagem do estudo } \\
\hline Quantitativo & 04 & 40 \\
\hline Qualitativo & 05 & 50 \\
\hline Quantitativo-Qualitativo & 01 & 10 \\
\hline \multicolumn{3}{|l|}{ Delineamento da pesquisa } \\
\hline Revisão Sistemática & 03 & 30 \\
\hline Estudo de Coorte & 04 & 40 \\
\hline Caso-Controle & 03 & 30 \\
\hline \multicolumn{3}{|l|}{ Idioma } \\
\hline Inglês & 08 & 80 \\
\hline Português & 01 & 10 \\
\hline Espanhol & 01 & 10 \\
\hline \multicolumn{3}{|l|}{ Nível de evidência } \\
\hline 01 & 03 & 30 \\
\hline 03 & 04 & 40 \\
\hline 04 & 03 & 30 \\
\hline \multicolumn{3}{|l|}{ Grau de Recomendação } \\
\hline A & 05 & 50 \\
\hline B & 05 & 50 \\
\hline \multicolumn{3}{|l|}{ Distribuição temporal } \\
\hline 2019 & 02 & 20 \\
\hline 2018 & 03 & 30 \\
\hline 2017 & 02 & 20 \\
\hline 2016 & 02 & 20 \\
\hline 2015 & 01 & 10 \\
\hline \multicolumn{3}{|l|}{ Procedência } \\
\hline EUA & 06 & 60 \\
\hline Brasil & 01 & 10 \\
\hline Índia & 01 & 10 \\
\hline Irã & 01 & 10 \\
\hline Espanha & 01 & 10 \\
\hline
\end{tabular}

Fonte: Elaboração própria (2020).

Dos dez artigos incluídos nesta revisão, oito estavam na linga inglesa (80\%), um na língua portuguesa (10\%) e um na língua castelhana (10\%). A maioria das publicações foi concentrada no ano de 2018 (30\%); em 2019 foram publicados dois artigos (20\%). A maioria dos artigos fez a utilização da abordagem qualitativa. Os estudos foram organizados conforme títulos, autores, objetivo principal e perfil amostral (Quadro 2). 
Quadro 2. Publicações incluídas segundo o título do artigo, autor, objetivo principal e perfil amostral. Caxias - MA, 2020. (N=10).

\begin{tabular}{|c|c|c|c|c|}
\hline $\begin{array}{l}\mathbf{N}^{\circ} \text { DE ORDEME } \\
\text { BASE }\end{array}$ & TÍTULO DO ARTIGO & AUTORES & OBJETIVO PRINCIPAL & PERFIL AMOSTRAL \\
\hline $\begin{array}{c}\text { A1 } \\
\text { BIREME }\end{array}$ & $\begin{array}{l}\text { Spirituality and } \\
\text { Interprofessional } \quad \text { Healthcare } \\
\text { Education: AnExploratory Study. }\end{array}$ & $\begin{array}{c}\text { Hovland, } \\
\text { Niederriter e } \\
\text { Thoman, (2018) }\end{array}$ & $\begin{array}{l}\text { O objetivo do estudo foi compreender as percepções } \\
\text { de estudantes da área da saúde (enfermagem, serviço } \\
\text { social, farmácia, aconselhamento de saúde e } \\
\text { aconselhamento escolar) em relação à espiritualidade. }\end{array}$ & $\begin{array}{l}\text { Coleta de dados ocorreu em dois pontos no } \\
\text { tempo, com duas amostras diferentes com } 131 \\
\text { alunos no total }(65 \mathrm{em} 2015 ; 66 \mathrm{em} 2016) \text {. }\end{array}$ \\
\hline $\begin{array}{c}\text { A2 } \\
\text { BIREME }\end{array}$ & $\begin{array}{l}\text { Percepção de Acadêmicos de } \\
\text { Medicina e de Outras Áreas da } \\
\text { Saúde e Humanas (Ligadas à } \\
\text { Saúde) sobre asRelações entre } \\
\text { Espiritualidade, Religiosidade e } \\
\text { Saúde }\end{array}$ & Ferreira et al.(2018) & $\begin{array}{l}\text { Analisar o pensamento dos acadêmicos ingressantes } \\
\text { no curso de Medicina e de outras áreas da saúde e } \\
\text { humanas acerca do tema; identificar o papel e a } \\
\text { importância da religiosidade e espiritualidade em suas } \\
\text { vidas e futuras práticas profissionais. }\end{array}$ & $\begin{array}{l}270 \text { alunos de } 18 \text { a } 43 \text { anos; entretanto, } 183 \\
\text { preencheram o questionário, sendo a maioria } \\
\text { do sexo feminino, de família convencional, } \\
\text { cristã/ católica, que herdou a religião dos pais; } \\
78,6 \%(144 / 183) \text { referiram afiliação religiosa, } \\
43,5 \% \text { (87/183) a exercem de forma } \\
\text { organizacional. }\end{array}$ \\
\hline $\begin{array}{c}\text { A3 } \\
\text { BIREME }\end{array}$ & $\begin{array}{l}\text { Public, patient and carers' views } \\
\text { on palliative and end-of-life care } \\
\text { in India }\end{array}$ & $\begin{array}{c}\text { Venkatasalu et al. } \\
\text { (2018) }\end{array}$ & $\begin{array}{l}\text { Rever a evidência existente sobre o público indiano, } \\
\text { paciente e cuidadores na perspectiva "sobrecuidados } \\
\text { paliativos e de fim de vida". }\end{array}$ & $\begin{array}{l}\text { Revisão integrativa em que foi realizada em } \\
\text { cinco bancos de } \\
\text { dados, usando pesquisas } \\
\text { manuais de periódicos importantes e } \\
\text { rastreamento de citações de referência para } \\
\text { estudos empíricos publicados } \\
\text { em inglês de } 1990 \text { a } 2015\end{array}$ \\
\hline $\begin{array}{c}\text { A4 } \\
\text { BIREME }\end{array}$ & $\begin{array}{lrr}\text { Qualitative } & \text { Analysis of } & \text { Faith } \\
\text { Community } \quad \text { Nurse- } & \text { Led } \\
\text { Cognitive-Behavioral } & \text { and } \\
\text { Spiritual Counseling } & \\
\text { for Dementia Caregivers. } & \end{array}$ & $\begin{array}{l}\text { Kazmer, } \\
\text { Glueckauf, } \\
\text { Schettini, Ma e } \\
\text { Silva (2018). }\end{array}$ & $\begin{array}{l}\text { Avaliar a efetividade de um sistema cognitivo com } \\
\text { intervenção de aconselhamento comportamental e } \\
\text { espiritual (CBSC) sobre mudanças na depressão e a } \\
\text { gravidade dos problemas de cuidado identificados e } \\
\text { problemas de autocuidado. }\end{array}$ & $\begin{array}{l}\text { Oito profissionais da saúde (todas mulheres) } \\
\text { participaram do programa e de um workshop } \\
\text { de treinamento de } 2,5 \text { dias. }\end{array}$ \\
\hline $\begin{array}{c}\text { A5 } \\
\text { BIREME }\end{array}$ & $\begin{array}{l}\text { Reasons behind providing futile } \\
\text { medical treatments in Iran. }\end{array}$ & $\begin{array}{l}\text { Aghabarary e } \\
\text { Nayeri (2017) }\end{array}$ & $\begin{array}{l}\text { Explorar enfermeiras e médicos iranianos sobre a } \\
\text { percepções das razões por trás de tratamentosmédicos } \\
\text { fúteis. }\end{array}$ & $\begin{array}{l}\text { Vinte e um enfermeiros } \\
\text { enove especialistas médicos foram recrutados } \\
\text { propositadamente em quatro hospitais de } \\
\text { ensino afiliados } \\
\text { Un à } \\
\text { Universidade de Ciências Médicas de Teerã. }\end{array}$ \\
\hline $\begin{array}{c}\text { A6 } \\
\text { BIREME }\end{array}$ & $\begin{array}{l}\text { Chaplains on the MedicalTeam: } \\
\qquad \mathrm{A} \text { Qualitative } \\
\text { Analysis of an } \\
\text { Interprofessional Curriculum for } \\
\text { InternalMedicine Residents and } \\
\text { Chaplain Interns. }\end{array}$ & $\begin{array}{l}\text { Hemming, Teague, } \\
\text { Crowe eLevine } \\
\text { (2016) }\end{array}$ & $\begin{array}{l}\text { Identificar os benefícios e desafios percebidos e } \\
\text { vivenciados pelos participantes do estudo. Os grupos } \\
\text { focais incluíram médicos internos supervisionando } \\
\text { médicos assistentes, estagiários e residentes de } \\
\text { medicina interna. }\end{array}$ & $\begin{array}{l}\text { Utilizou-se seis grupos focais separados entre } \\
\text { dezembro de } 2012 \text { e maio de 2015: um com } \\
\text { médicos assistentes, três com grupos de } \\
\text { estagiários, e dois com médicos residentes. }\end{array}$ \\
\hline A7 & La formación en & Cordero, Castillo e & conveniência & Uma revisão da literatura científica dos \\
\hline
\end{tabular}




\begin{tabular}{|c|c|c|c|c|}
\hline CINAHL & $\begin{array}{l}\text { religiosidade } y \\
\text { espiritualidad en losestudios de } \\
\text { Grado en } \\
\text { Enfermería. }\end{array}$ & Escãno (2019) & $\begin{array}{l}\text { religiosidade/espiritualidade nos planos de estudo do } \\
\text { grau de enfermagem. }\end{array}$ & $\begin{array}{l}\text { últimos } 5 \text { anos (2012-17) foi realizada nos } \\
\text { bancos de dados Cinahl, Scopus, Pubmed e } \\
\text { Web of Science (WOS). }\end{array}$ \\
\hline $\begin{array}{c}\text { A8 } \\
\text { PUBMED }\end{array}$ & $\begin{array}{l}\text { Meaning Making and Religious } \\
\text { EngagementAmong Survivors of } \\
\text { Childhood Brain Tumors and } \\
\text { Their Caregivers }\end{array}$ & $\begin{array}{c}\text { Rabelais, Jones, } \\
\text { Ulrich e Deatrick } \\
\text { (2019) }\end{array}$ & $\begin{array}{l}\text { Descrever como adolescentes e jovens adultos } \\
\text { sobreviventes e suas mães-cuidadoras atribuem } \\
\text { significado à sua experiência de sobrevivência a } \\
\text { tumores pós-cerebrais, com foco nos benefícios das } \\
\text { descobertas e interseções com envolvimentoreligioso }\end{array}$ & $\begin{array}{l}\text { Adolescentes e jovens adultos sobreviventes } \\
\text { de tumores cerebrais na infância e suas } \\
\text { famílias, vivendo em ambientes } \\
\text { comunitários. Entrevistas individuais e } \\
\text { semiestruturadas simultâneas e separadas das } \\
40 \text { díades correspondentes ( } 80 \text { entrevistas } \\
\text { totais) ocorreu usando a análise de conteúdo } \\
\text { convencional entre e dentro das díades. }\end{array}$ \\
\hline $\begin{array}{c}\text { A9 } \\
\text { PUBMED }\end{array}$ & $\begin{array}{l}\text { Integrating chronic kidneydisease } \\
\text { patient's } \\
\text { spirituality in their care: health } \\
\text { benefits and } \\
\text { research perspectives }\end{array}$ & $\begin{array}{l}\text { Fradelos et al. } \\
\qquad(2015)\end{array}$ & $\begin{array}{l}\text { O objetivo deste artigo é examinar evidências da } \\
\text { literatura internacional sobre a possível relação entre } \\
\text { espiritualidade e resultados de saúde, principalmente } \\
\text { no complexo códice de uma doença crônica e de } \\
\text { vida, como a DRC. }\end{array}$ & Pacientes com Doença Renal Crônica. \\
\hline $\begin{array}{c}\text { A10 } \\
\text { PUBMED }\end{array}$ & $\begin{array}{l}\text { Patient and Healthcare } \\
\text { Perspectives on the Importance } \\
\text { and Efficacy of Addressing } \\
\text { Spiritual Issues Within an } \\
\text { Interdisciplinary Bone } \\
\text { Marrow Transplant Clinic: A } \\
\text { Qualitative Study. }\end{array}$ & Shane (2016) & $\begin{array}{l}\text { Entender melhor a importância e a eficácia do } \\
\text { tratamento de questões espirituais em uma clínica } \\
\text { interdisciplinar de transplante de medula óssea, na } \\
\text { perspectiva de pacientes e profissionais de saúde. }\end{array}$ & $\begin{array}{l}\text { Grupos focais foram realizados com pacientes } \\
(\mathrm{n}=7) \text { e profissionais de saúde }(\mathrm{n}=9) \text { para } \\
\text { explorar a importância de abordar questões } \\
\text { espirituais ao longo da trajetória de tratamento } \\
\text { e identificar fatores associados ao atendimento } \\
\text { eficaz dessas necessidades. }\end{array}$ \\
\hline
\end{tabular}

Fonte: Pesquisa Direta (2020). 
Os estudos abordavam sobre as percepções dos profissionais frente à espiritualidade, à efetividade de programas de espiritualidade na prática clínica, aos principais desafios na implementação da espiritualidade na prática clínica e às percepções dos acadêmicos sobre a espiritualidade e o cuidado.

\section{Discussão}

\section{Os benefícios das práticas religiosas na prestação de cuidados de enfermagem}

A espiritualidade do paciente é vista como componente essencial para o processo de cura do indivíduo; ao assistencial os pacientes e familiares com uma abordagem espiritual-religiosa emprega-se a "filosofia cultural". Os pacientes precisam de um suporte significativo e satisfatório, além de práticas médicas, pois um dos objetivos mais benéficos da espiritualidade é o autocuidado da equipe para evitar o desgaste (Hovland, Niederriter, \& Thoman, 2018).

Os fundamentos de cuidados aos doentes nas comunidades cristãs no passado, represrntou uma grande influência para a construção de hospitais e formações de profissionais de saúde. A fé se apresentou como uma estratégia necessária para o enfrentamento de diversas alterações de vulnerabilidade física e psíquica dos pacientes, por isso a necessidade de uma abordagem entre a ciência, religiosidade e espiritualidade na prática do cuidado (Ferreira et al., 2018).

A futilidade médica envolve fatores diversificados como objetivo terapêutico, questões emocionais, pessoais, contexto religioso e sociocultural e valores dos profissionais de saúde, englobando o paciente e a família. Tendo com intuito de prolongar a vida e proporcionar aos pacientes maior conforto físico, emocional e a proteção da dignidade (Aghabarary \& Nayeri, 2017).

As famílias se aproximam de uma presença divina para sentir o conforto e consolo em seu tratamento, buscando fortalecimento psicológico e social para continuar o tratamento e encontrar um significado para a vida (Rabelais, Jones, Ulrich, \& Deatrick, 2019).

A dimensão espiritual deve ser implementada na enfermagem. A pioneira Florence Nightingale observou ser humano com olhar holístico, reconhecendo a dimensão espiritual na assistência de enfermagem como uma das fontes essenciais de cura. De acordo com o Conselho Internacional de Enfermagem, esta é responsável por avaliar e fornecer cuidado holístico, atribuidos necessidades espiritual e religiosa (Fradelos et al., 2015).

O bem-estar espiritual afeta de maneira positiva as condições de vida, o enfrentamento, a resistência, o significado da vida e a superação pós-trauma. A fé, a oração e a cura espiritual são os recursos alternativos mais utilizados pelos pacientes, proporcionando um impacto positivo diante dos problemas psicossociais, incluindo pressão reduzida, e mostrando ótimos resultados de saúde (Shane, 2016).

Assistência de enfermagem em conjunto com os cuidados espirituais é fundamental para o bem-estar mental, a valorização do indivíduo, aceitação da patologia, pois o paciente adquire esperança e se sente mais resistente, enquanto a equipe de enfermagem se ver satisfeita por prestar cuidados humanizado, emoções positivas e aumenta a qualidade de vida no trabalho (Cordero, Castillo, \& Escaño, 2019).

\section{A importância da espiritualidade no estado de saúde}

A espiritualidade é caracterizada como aspectos emocionais e otimista, apoiados por considerações cognitivas nas quais ajudam o paciente passar por eventos individuais em seu processo de estabilidade e cura. Enquanto que a religião é um conjunto organizado de regras, práticas, crenças, valores e limites estabelecidos por um Poder Superior, aderidos pelos indivíduos. Na enfermagem a espiritualidade e a religiosidade torna a assistência mais humanizada e aproxima o profissional do paciente (Hovland et al., 2018).

O termo "espiritualidade baseada em evidências" ao longo de décadas acumularam registros de que as atividades espirituais e religiosas influenciassem positivamente inúmeros aspectos da saúde humana, sendo que o cuidado em saúde no ser 
humano deve aborda-lo como uma unidade biopsicossocial, levando em consideração os aspectos biológicos, sociais e psicológicos, e acrescentando a espiritualidade (Ferreira et al., 2018).

Os pacientes veem a espiritualidade como uma conexão com sagrado, como a natureza e com o eu, buscando assim o significado e propósito para a vida. A espiritualidade é algo muito importante para os pacientes, os quais afirmam sentir um conforto e confiança quando conectam suas crenças religiosas aos cuidados médicos (Hemming, Teague, Crowe, \& Levine, 2016).

Geralmente após um tratamento eficiente ou uma reavaliação positiva de quadro clínico os pacientes e seus cuidadores traduzem esses resultados como uma respota significativa ligada às crenças e ao significado de valorização da vida (Rabelais, Jones, Ulrich, \& Deatrick, 2019).

A enfermagem exige um cuidado holístico, sua ciência reconhece que o ser humano possui uma dimensão espiritual, dentro desta dimensão a respeito, temor, inspiração e o propósito da vida. A dimensão espiritual é essencial nos processos de curas, doença física, estresse emocional, luto e morte. Entrar em consenso com bem-estar espiritual tornará o processo de cura mais eficiente (Fradelos et al., 2015).

\section{As principais limitações e desafios nos cuidados de enfermagem com as práticas de cura}

Pacientes relatam que geralmente utilizam a conexão espiritual com a assistência médica simultaneamente o que aumenta a qualidade e o prazer da vida. A falta de conceitos de espiritualidade no currículo para os estudantes de enfermagem ao apoio espiritual faz enfermeiros se sentirem desconfortáveis ao discutirem atividades espirituais (Hovland et al., 2018).

Os cuidados das áreas da saúde estão baseados em uma concepção mecanicista do homem, em que estabelece uma assistência precária de humanização, a carência de espiritualidade e religiosidade na assistência são fortemente relatadas nas pesquisas (Ferreira et al., 2018).

As novas estratégias de cuidados trazem medo aos pacientes e aos cuidadores, geralmente, são familiares, tendo como barreiras socioculturais, geográficas e financeiras. Os pacientes tendem a desconfiar e ter medo de novas estratégias e intervenções fora de seu local, principalmente quando o tratamento não contempla seus valores socioculturais e religiosos o que leva a conflitos e não adesão das intervenções (Kazmer, Glueckauf, Schettini, Ma, \& Silva, 2017).

Um estudo realizado no Irã, focado nos tratamentos que contemplam a espiritualidade e a religiosidade, identificou que essa linha de cuidados está relacionada estreitamente com os princípios éticos de autonomia, beneficência, não maleficência e justiça. Esses tratamentos e cuidados são levados em conta no final da vida, contudo, há desafios para a sua implementação pela equipe médica e especialistas (Aghabarary \& Nayeri, 2017).

Há uma forte carência nas relações dos capelães com os médicos: ambos possuem uma compreensão limitada um do outro, ocasionando um déficit na assistência de cuidados ao paciente. A equipe médica apresenta uma dificuldade ao atendimento às necessidades espirituais do paciente em comparação a outros membros da área da saúde. Os capelães apresentam um desconforto na conexão dos médicos com a assistência do paciente (Hemming et al., 2016).

Quando a equipe de saúde desconsidera na assistência as necessidades religiosas do indivíduo, torna-se um obstáculo establecer um vínculo com esse paciente, pois ocasiona na perde de confiançae na sensação de insegurança no indivíduo. Enquanto que, se no cuidado tiverem considerando as práticas e necessidades religiosas do paciente, isso promove uma maior participação do mesmo no tratamento, e consequentemente mais eficiência, pois o indidividuo se sentirá seguro e confortável (Rabelais et al., 2019).

É desafiador manter a assistência pelos profissionais da saúde quando é preciso abordar questões espirituais, necessitando de apoio especializado em cuidados espirituais (Shane, 2016). A falta de implementação na grade curricular de enfermagem caracteriza alguns fatores como a insegurança dos estudantes de enfermagem, em prestar cuidados espirituais, a 
falta de conhecimento nesse campo, a falta de compreensão da espiritualidade na prática (Cordero et al., 2019).

\section{Condutas de enfermagem correlacionadas com o tratamento elou terapia cultural religiosa}

A enfermagem deve considerar a espiritualidade como um componente fundamental para a saúde do paciente, estando presente na avaliaçãocom holística do profissional estabelecendo assim confiança e credibilidade no atendimento prestado (Hovland et al., 2018).

No caso de pacientes terminais a assistência de enfermagem deve explorar as necessidades físicas, espirituais, psicossociais do indivíduo, estando atenta a preferência do local da morte, a situação domiciliar do paciente e as necessidades informais dos familiares, como a utilização de mantras, água benta, amuletos, fotos de seu Deus; geralmente utilizados como forma de receber força em períodos de sofrimento (Venkatasalu et al., 2018).

O desenvolvimento e a implementação de estratégias pelos enfermeiros e cuidadores devem abordadr as características culturais do paciente, incorporando a espiritualidade, e baseando-se na fé, o que muitas vezes representa uma quebra de barreiras entre o profissionals e religiosidade/espiritualidade, mas que demonstra uma prática humanizada e holística na contrução do plano de cuidados, e que melhora a comunicação e o relacionamento interpessoal entre o profissional e o paciente (Kazmer et al., 2018).

Uma equipe estruturada e que ututiliza a assistência espitritual na prática, é uma equipe que respeita o paciente e seus valores, bem como suas crenças, e isso é essêncial para o sucesso e credibilidade da equipe na prestação de cuidados. O ensino da espiritualidade no atendimento a paciente, e, a educação interprofissional em saúde apresenta benefícios para uma abordagem interprofissional, educacional em níveis de cuidados em contextos diversificados. (Hemming et al., 2016).

Planejar e implementar uma assistência de enfermagem abordando de maneira significativa e respeitosa, a espiritualidade do paciente contribui para a melhoria do bem-estar psicológico, biológico e espiritual do indivíduo. A espiritualidade promove mudanças no estilo de vida, e também pode influenciar no comportamento, e no estado de saúde (Fradelos et al., 2015).

\section{Conclusão}

A enfermagem e outras áreas da saúde devem observar o ser humano em seus aspectos sociais, culturais, religiosos e espirituais. Atendendo e prestando uma assistência de saúde humanizada, o processo de cura e o processo de reabilitação se mostram bastantes eficazes no estado de saúde do indivíduo, quando estão em conjunto com as necessidades espiritual/religiosa. Percebe-se que a religiosidade e espiritualidade proporcionam uma confiabilidade, fortalecimento e encorajamento no tratamento do paciente. Assim, a equipe de enfermagem se sente satisfeita no processo de humanização, e consequentemente também aumenta a qualidade de vida do enfermeiro.

No decorrer da pesquisa, uma das limitações encontrada foi a escassez de artigos sobre a temática, fazendo com que as estratégias de busca nas bases de dados fossem frequentemente alteradas, até serem apresentados artigos que se enquadrassem na temática abordada.

Abordar a espiritualidade e religiosidade na área da saúde é fundamental para aprimorar as estratégias de cuidados com foco na visão holística, por isso ainda é necessário explorar essa temática e desenvolver estudos que contemplem aespiritualidade, religiosidade e sua relação nos cuidados de enfermagem, e também no relacionamento cliente e profissional, podendo também ser utilizada para melhorar a eficiência da assistência utilizando todas as dimensões essenciais para a saúde do ser humano.

Este estudo analisou os desafios e limitações na implementação da espiritualidade e religiosidade pelos enfermeiros, espera-se que a temática promova debates e pensamentos críticos, bem como estimular o desenvolvimento de estratégias que adaptem os cuidados de forma integral as necessidades dos pacientes, e reconhecer que a associação dos cuidados de saúde nos 
âmbitos espirituais e religiosos visibilizam assistência ao indivíduo como uma unidade biopsicossocial, sendo assim um dos pilares para a saúde.

\section{Referências}

Aghabarary, M. \& Nayeri, N. D. (2017). Reasons behind providing futile medical treatments in Iran. Nurs Ethics., 24(1),33-45.

Alves, D.A., Silva, L.G., Delmondes, G. A., Lemos, I. C. S., Kerntopf, M. R., \& Albuquerque, G. A. (2016). Cuidador de criança com câncer: religiosidade e espiritualidade como mecanismos de enfrentamento. Revista Cuidarte, 7(2),1318-24.

Bork, A M T. (2011). Enfermagem baseada em evidencias. Guanabara Koongan.

Cervelin, A. F. \& Kruse, M. H. L. (2014). Espiritualidade e religiosidade nos cuidados paliativos: conhecer para governar. Escola Anna Nery Revista de Enfermagem, 18 (1),136-142.

Cordero, R. D., Castillo, C. S., \& Escaño, J. V. (2019). La formación en religiosidad y espiritualidad en los estudios de Grado en Enfermería. Cult. Cuid, 23 (53), 304-315.

Crossetti, M. G. O. (2012). Revisão integrativa de pesquisa na enfermagem o rigor cientifico que lhe é exigido. Rev Gaúcha Enferm., 33(2), 8-9.

Ercole' F. F., Melo, L. S., Alcoforado, C. L. G. C. (2014). Revisão integrativa versus revisão sistemática. Rev Min Enferm (REME), $18(1), 1-260$.

Ferreira, A. G. C., Duarte, T. M. M., Silva, A. F., \& Bezerra, M. R. (2015). Concepções de espiritualidade e religiosidade e a prática multiprofissional em cuidados paliativos. Revista Kairós: Gerontologia, 18 (3), 227-244.

Ferreira, T.T.F., Borges, M. F., Zanetti, G. C., Lemos, G. L., Gotti, E. D., Tomé, J. M., \& Rezende, E. A. M. R. (2018). Percepção de Acadêmicos de Medicina e de Outras Áreas da Saúde e Humanas (Ligadas à Saúde) sobre as Relações entre Espiritualidade, Religiosidade e Saúde. Rev. Bras. Educ. Méd., 42 (1), 67-74.

Fradelos, E. C., Tzavella, F., Koukia, E., Papathanasiou, I.V., Alikari, V., Stathoulis, J., \& Zyga, S. (2015). Integrating Chronic Kidney Disease Patient's Spirituality In Their Care: Health Benefits And Research Perspectives. Mater Sociomed., 7(5), 354-358.

Hemming, P., Teague, P. J., Crowe, T., \& Levine, R. (2016). Chaplains on the Medical Team: A Qualitative Analysis of an Interprofessional Curriculum for Internal Medicine Residents and Chaplain Interns. J Relig Health, 55 (2), 560-71.

Hovland, C., Niederriter, J., \& Thoman, J. (2018). Spirituality and Interprofessional Healthcare Education: An Exploratory Study. J Christ Nurs., 35 (4), e47e52.

Kazmer, M. M., Glueckauf, R. L., Schettini, G., Ma , J., \& Silva, M. (2018). Qualitative Analysis of Faith Community Nurse-Led Cognitive- Behavioral and Spiritual Counseling for Dementia Caregivers. Qual Health Res., 28 (4), 633-647.

Melo, C. F., Sampaio, I. S., Souza, D. L. A., \& Pinto, N. S. (2015). Correlação entre religiosidade, espiritualidade e qualidade de vida: uma revisão de literatura. Estudos e Pesquisas em Psicologia, 15 (2), 447-464.

Nascimento, L. C., Santos, T. F. M., Oliveira, F. C. S., Pan, R., Santos, M. F., \& Rocha, S. M. M. (2013). Espiritualidade e religiosidade na perspectiva de enfermeiros. Texto Contexto Enferm, 22 (1), 52-60.

Oliveira, A. L. C. B., Feitosa, C. D. A., Santos, A. G., Lima, L. A. A., Fernandes, M. A., \& Monteiro, C. F. S. (2017). Espiritualidade e religiosidade no contexto do uso abusivo de drogas. Rev Rene, 18 (2), 283-90.

Oliveira, F. B. M., Costa, A. C. A. L., Alves, D. L., França, J. F., Macedo, M. S., \& Santos, R. D. (2016). Relação entre a sobrecarga de trabalho e erros de administração de medicação na assistência hospitalar. Revista Ciências \& Saberes, 2(2), 325-334.

Pompeo, D. A., Rossi, L. A., Galvão, C. M. (2009). RevisÃo integrativa: etapa inicial do processo de validação de diagnóstico de enfermagem. Acta Paul Enferm., 22(4):434-8.

Rabelais, E., Jones, N. L., Ulrich, C. M., \& Deatrick, J. A. (2019). Meaning making and religious engagement among survivors of childhood brain tumors and their caregivers. Oncol Nurs Forum., 46 (2), 170-18.

Shane, S. (2015). Patient and Healthcare Perspectives on the Importance and Efficacy of Addressing Spiritual Issues Within an Interdisciplinary Bone Marrow Transplant Clinic: A Qualitative Study. BMJ Open, 5 (11).

Venkatasalu, M. R., Jagadeesh, N. S., Elavally, S., Pappas, Y., Mhlanga, F., \& Varatharajan, R. P. (2018). Public, patient and carers' views on palliative and end-of-life care in India. Int Nurs Rev., 65 (2), 292-301. 